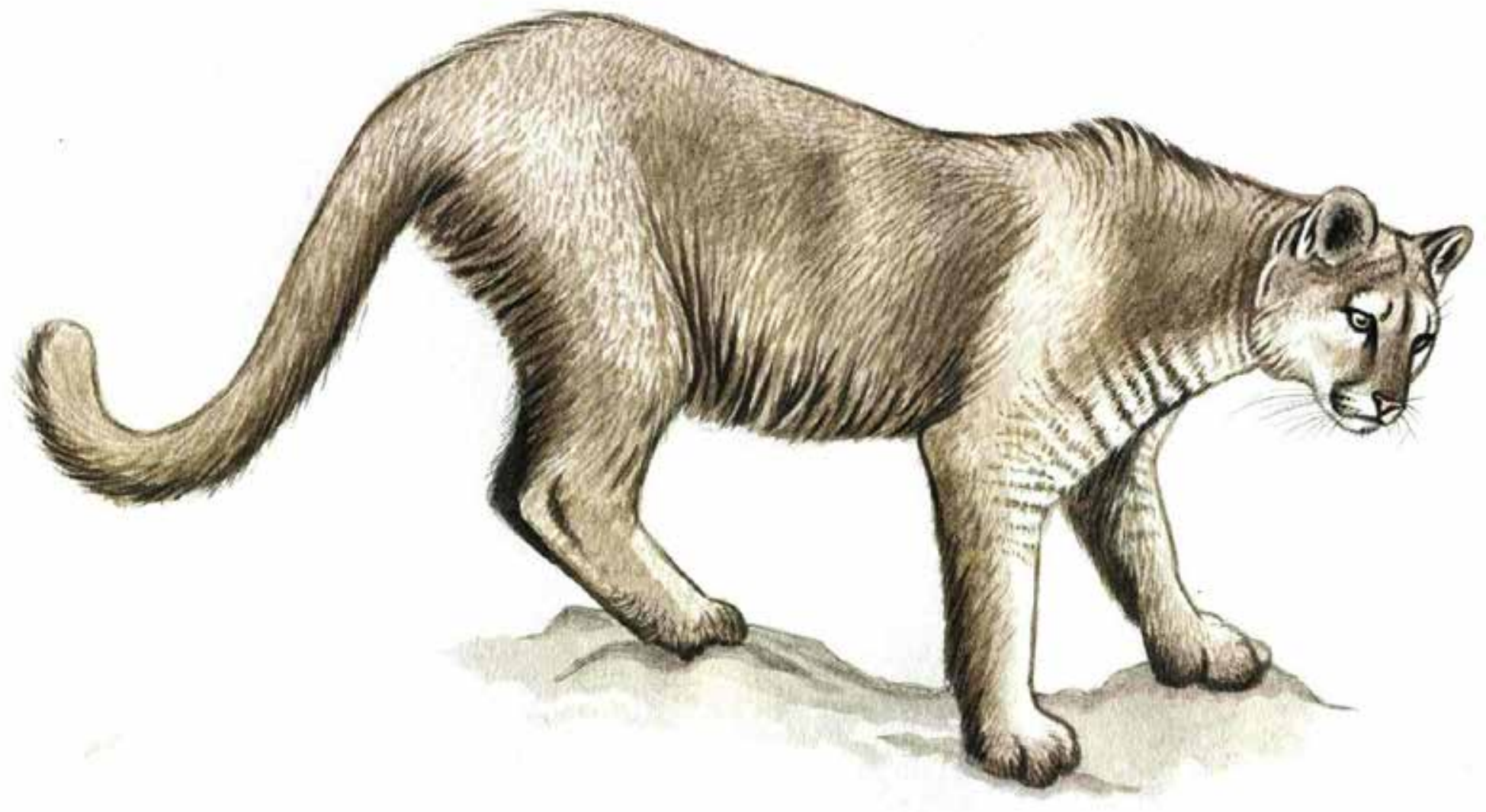

Leonardo González Galli

Profesor Investigador Univesidad de Buenos Aires

Técnica: Acrílico sobre Cartón 


\section{POSIBILIDADES E IMPOSIBILIDADES DE LA INCORPORACIÓN DE LA EDUCACIÓN AMBIENTAL EN LA ESCUELA:PROPUESTA DE ESTRATEGIA EVALUATIVA}

\section{Impossibilities and Possibilities about Incorporation of Environmental Education in the School: Evaluative Strategy Proposal}

Fecha de recepción: 14 de marzo de 2014

Fecha de aprobación: 13 de junio de 2014

Carolina Vargas Niño ${ }^{1}$

\section{Resumen}

El propósito de la investigación fue formular una estrategia para evaluar los alcances de la incorporación de la propuesta de transversalidad de la educación ambiental (EA) en el currículo de la escuela, a la luz de las posibilidades o la imposibilidades que los maestros rescatan en su quehacer y ser, dentro de sus realidades escolares, con el objetivo de contrastar los hallazgos, a través de categorías conceptuales, metodológicosy operativos desde la Política Nacional de Educación Ambiental (PNEA), como panorama de lectura y comprensión de una realidad educativa que responde a la normatividad del sistema gubernamental nacional e internacional, al aproximarse a la resolución de las problemáticas ambientales y al alcance del desarrollo sostenible. La indagación se proyecta a la comprensión de las nociones que tienen los maestros en ejercicio de la forma como se está llevando a cabo la incorporación de la EA en sus instituciones, mediante la técnica de recolección de información de grupo focal. Se llegó así a concluir que el saber ambiental dentro de la escuela, se evidencia como aquel que está orientado desde la necesidad por el "hacer" por un "activismo" acelerado, que intenta más allá de la comprensión de las dinámicas ambientales, minimizar el desconocimiento del daño ambiental y propender por un estado de conservación de los recursos, en donde se expone la relación con el concepto de ambiente, como recurso para utilizar y proteger.

\section{Palabras clave}

Educación ambiental, escuela, estrategia evaluativa, Política Nacional de Educación Ambiental.

1 Profesora del Departamento de Biología. Universidad Pedagógica Nacional, magíster en Educación; coinvestigadora de la línea de Investigación "Biodiversidad y Conservación de los Ecosistemas Acuáticos de la Región Andina (SARA)".

Correo electrónico: Carov_1008@hotmail.com 


\section{Abstract}

The purpose of the research was to develop a strategy to evaluate the reaches of the incorporation of the proposal of transversal environmental education in the school curriculum, in light of the possibilities or impossibilities that teachers rescue in their performance and being in their educational situations, in order to contrast the findings, through conceptual, methodological and operational categories from the National Policy of Environmental Education as panorama of reading and understanding of educational reality which meets the regulations of national and international governmental system approaching the resolution of environmental problems and achieving sustainable development. The inquiry is projected to the understanding about notions that teachers are practicing and the way the incorporation of environmental education in their institutions is being conducted, using the technique "Focus Group" to collect the information. As a conclusion it is said that environmental knowledge within the school can be seen as one guided from the need of " to do" by "an accelerated activism" which tries to understand environmental dynamics more than minimize the unknowledge of environmental damage and to work for a statement of conservation of resources, where the relationship with the environmental concept is shown as a resource to be used and protected.

\section{Keywords}

Environmental Education, school, evaluative strategy, National Environmental Education Politics

\section{Introducción}

En la actualidad el discurso ambiental ha cobrado importancia a partir del reconocimiento de diversas dificultades de carácter ambiental en los ámbitos global y local, en donde el concepto de problemática ambiental surge de la manera en que una sociedad se vincula con la naturaleza para construir su hábitat y generar su proceso productivo y reproductivo, y está determinada por un sinnúmero de relaciones cuya práctica involucra desde la manera en que el hombre se concibe a sí mismo como parte de la naturaleza, hasta los instrumentos concretos con que se apropia de ella (Morin, 1999).

Esto se refiere a la amplitud de los diferentes saberes y prácticas tanto económicos como sociales y culturales de la humanidad, define además las características de configuración como grupo social y de la necesidad de suplir y desarrollar estrategias para que la adaptación a un espacio en particular habitable se conviertan cada vez más en un símbolo de una "evolución" óptima, si se puede llamar así, a la urgencia de ir hacia delante en la conquista del mundo y de sus recursos para vivirlo cómodamente.

No obstante, el análisis de esas prácticas y comportamientos de los grupos sociales dejan un panorama turbio en cuanto a la relación armónica entre la naturaleza del recurso ambiental y la naturaleza racional del hombre, que inconsciente lo utiliza, comprendiendo que tal vez esa inconsciencia-definida como la ausencia de conocer responsablemente lo que se hace y se quiere hacer-, pudo llegar a ser el punto de partida de las discusiones que sobre las problemáticas ambientales se han generado, y de lo que es peor, de una encrucijada de la que aún no se ha logrado salir. Dicha conciencia tomó el principio y el final de la temática académica y práctica del discurso ambiental, reduciéndolo casi a un absurdo difícil de alcanzar por quien biológica y socialmente puede transformarla: el hombre.

La polémica que se genera por cada uno de los eventos que resultan de las problemáticas ambientales han sido tema de diversas convenciones mundiales como la Conferencia de las Naciones Unidas sobre el Medio Humano, celebrada en Estocolmo en 1972, la cual es considerada un hito fundamental, donde se trató la problemática ambiental, cuáles son las causas, las consecuencias y sus posibles soluciones a la crisis del medio ambiente. Calificada como el primer esfuerzo internacional para enfrentar los problemas ambientales sobre una base global, dejó claro que las ideas de la sociedad referentes al planeta en que se vive y de cómo se podrá subsistir en él, estaban cambiando de modo radical (Castro, 1997).

Ahora bien, el cambio parece haber suscitado dos posiciones (Maya, 1992): una desde la generación de la angustia, de la desesperanza por perder algo que ya se consideraba ganado, propio e inagotable: el recurso ambiental, y la otra, desde una preocupación sin proyección por humanizar al hombre para que cuidara y conservara el recurso para las actuales y próximas generaciones, es decir, aún se puede utilizar si se logra conservar.

El ambiente se convierte así en problema de investigación debido al deterioro de los recursos naturales, y al afectar la vida humana a grandes y pequeñas escalas, centrándose la atención de la comunidad científica internacional, en la búsqueda de la concienciación de la necesidad apremiante de utilizar responsablemente el saber de todos los campos de la ciencia para darle respuesta a la 
creciente degradación ambiental, que no solo pone en crisis las condiciones de vida en el planeta, sino hasta la permanencia de la vida en este.

En aquel instante el discurso ambiental pierde su lugar fuera de la conciencia del hombre; es decir, por necesidad se tiene que reconocer que el exterior es afectado por el hombre de manera directa y rápida, y que es solo desde aquel interior que podría llegar a transformar el problema en una solución. La ciencia y la tecnología académicamente se ocupan de ofrecer una magnitud de funciones y estrategias que pueden ser prácticas para sostener en un mediano plazo el deterioro ambiental, pero son las Ciencias Humanas y de la Educación a las cuales se les otorga la formación del hombre que se requiere para conservar el ambientey que debe transformarse en pensamiento, acción y comportamiento, desde su ser natural y aprender a relacionarse intrínsecamente con el ambiente que conforma (Otero, 2006).

De allí surge la educación ambiental (EA) como la posibilidad de resolver el problema que ya demostraba una pista: la relación entre el hombre y su ambiente, la responsabilidad de ser productivo conservando, la imposibilidad de la infinita existencia del recurso, y a su vez, la dura batalla en el proceso formativo del ser humano desde diferentes contextos económicos, políticos, sociales y culturales, que de alguna forma se deben concentrar en un solo objetivo, en un tiempo limitado por la existencia y la sensibilidad social.

En el ámbito escolar, son los maestros quienes determinan en gran medida la experiencia de vida de los estudiantes, son los directivos y administrativos de un sistema educativo quienes determinan la exigencia de productividad intelectual y económica de una nación política, y es en suma la sociedad quien adopta los comportamientos y formas de ver, pensar, utilizar y habitar el lugar.

No obstante el cómo debiese entenderse la EA y el qué debe enseñarse, junto con sus estrategias y fundamentos permanecen consignados en la PNEA, que sumado a los lineamientos dados por el Ministerio de Educación Nacional, decretan la tarea de educar en las instituciones educativas a los líderes, dinamizadores y gestores ambientales del país, con una proyección no solo local, nacional sino internacional, asegurando así el éxito en su tarea a partir de tan claras y bien redactadas pautas ambientales.

De acuerdo con el Decreto 1743 de 1994, que institucionaliza el Proyecto Ambiental Escolar (PRAE), "[...] surge la visión de encaminar esfuerzos para propiciar un cambio con respecto al manejo de los recursos en las comunidades locales, por ello con base en la Ley 99 de 1993 y la Ley 115 de 1994".
De esta forma se busca que desde la escuela se analicen las problemáticas ambientales con el propósito de generar espacios de reflexión, investigación y acción que permitan a la comunidad educativa contribuir al mejoramiento de la calidad de vida a través del respeto, la tolerancia, la participación la autonomía y la autogestión. Los PRAE son el espacio de investigación que se debe construir sólidamente en las escuelas, y que maestros, directivos y demás estamentos del sistema educativo deben hacer posible con la más alta calidad y eficiencia.

Ahora bien, si la EA es un espacio curricular que propone lineamientos y normas de formación personal y académica para los estudiantes, que se debe basar en la innovación, en la transformación de valores, actitudes y procesos de productividad, que ya vienen descritos, ¿por qué aún no se han logrado resultados que garanticen la solución a problemáticas ambientales locales, regionales o sectoriales? ¿Será que el compromiso va más allá de tratar de educar al estudiante con lo que debe hacer y pensar sobre su ambiente, sino, del contexto y de las posibilidades en el cual se desarrolla como sujeto? ¿Será que los maestros comprendieron la idea de educar para el ambiente, o educar ambiental o integralmente a sus estudiantes? ¿Es posible entonces que la academia y los valores se unan en un horizonte de responsabilidad social y ambiental? Es más, ¿aún es posible pensar en una educación diferenciada de la formación integral del sujeto? Si esto es así, ¿qué comprende la EA en la escuela?, ¿cómo se complementan las realidades de los sujetos con sus espacios?, y lo que es aún más inquietante, ¿cómo hace el maestro para lograr tan altos objetivos sin que, en su totalidad, dependan de su quehacer como profesional y ser humano?

Puede que exista una respuesta monosílaba a cada una de las anteriores inquietudes, o que ni siquiera se puedan clasificar como inquietudes porque ya se analizaron y tienen evidencias sustentables en la realidad, pero es a través de la enseñanza como se da la posibilidad de cuestionar las evidencias hasta encontrar otras que soporten o difieran y contrasten la comprensión de la realidad.

Por tanto, este es el escenario dispuesto a la reflexión y la continua transformación del pensamiento y quehacer del hombre; así, los profesionales de la educación inmersos en la realidad escolar -en el espacio de aula, en la comunicación con los profesionales en formación, en constante diálogo con pares que aún generan angustias e incertidumbres sobre los PRAE, que aún no comprenden el sentido y el significado de la PNEA, desde su nomenclatura hasta su exposición temática, y que por supuesto son humanos preocupados por su ambiente-, han generado que sea pertinente y viable el dedicar un proceso investigativo que produzca un espacio para el diálogo de saberes frente a la incorporación de la EA en las escuelas (Cano, 1998). 
El problema entonces es aún mayor que la crisis ambiental; parece estar centrado en la enseñanza, en la forma de introducir en el currículo una enseñanza transversal e interdisciplinar de la EA y, por ende, de las estrategias pedagógicas y didácticas para colocarla en marcha en la comunidad educativa. Precisamente, ¿cómo lograr resultados perdurables y eficaces en una jornada escolar tan reducida a la memoria de conceptos y a la disciplina de estudio que al desarrollo de habilidades y construcción de significado y sentido del saber?

Este panorama parece desolador y casi que inventado, pero es generado por la realidad escolar, que con su apretado tiempo y con toda la normatividad en el diseño de proyectos transversales, pierde la esencia y el significado para la formación profesional y personal de comprender una problemática, desarrollarla y, posiblemente, solucionarla. En estos tiempos caóticos, a maestros y estudiantes les sobrepasa el afán por cumplir con lo reglamentario, por la nota alta, por pasar al siguiente grado o escalafón, por la posición académica y por el prestigio social, y se han olvidado de la convivencia humana, de los valores, de la vida en familia, del respeto por la vida y por una digna oportunidad de vivirla.

Por ello surgió la idea de construir una estrategia evaluativa que señale posibilidades e imposibilidades en la experiencia de la incorporación de la EA en la escuela a partir del quehacer del maestro, desde los criterios conceptuales, metodológicos y operativos planteados por la PNEA en cuatro instituciones educativas de Bogotá; desde el llamado al maestro investigador, que se encuentra dispuesto a tomar de su realidad cercana las problemáticas de su comunidad en un determinado contexto y con el desarrollo de un proceso de investigación, construir conocimientos, reflexionarlo y proponer estrategias de solución, que se describe en los lineamientos normativos del MEN y de la PNEA, y lograría de esta forma, consolidar una experiencia investigativa con el actual documento que invite a los colegas docentes a reflexionar y analizar la viabilidad de sus vivencias como un verdadero conocimiento pedagógico que puede complementar el esfuerzo de la educación integral para una EA.

\section{Materiales y métodos}

El estudio se enmarcó en la investigación educativa pues su interés es la necesidad de comprender el proceso conceptual, metodológico y actitudinal, que sigue la puesta en marcha de la EA, como propósito pedagógico y de transformación de valores y forma de vida de una sociedad. Igualmente, se hizo un análisis interpretativo y crítico del proceso llevado en la articulación en la escuela, desde el paso trascendental de la labor del maestro de la formación integral de la sociedad y cómo esta se ve reflejada en las acciones y comportamientos; por consiguiente, se enmarca en una postura interpretativa, desde el método sociocrítico, y el paradigma constructivista.

El paradigma sociocrítico, de acuerdo con Arnal (1992), adopta la idea de que la teoría crítica es una ciencia social que no es puramente empírica ni interpretativa; sus contribuciones se originan, "de los estudios comunitarios y de la investigación participante" (p. 98). Se fundamenta en la crítica social con un marcado carácter autorreflexivo; considera que el conocimiento se construye siempre por intereses que parten de las necesidades de los grupos; pretende la autonomía racional y liberadora del ser humano; y se consigue mediante la capacitación de los sujetos para la participación y transformación social.

Utiliza la autorreflexión y el conocimiento interno y personalizado para que cada quien tome conciencia del rol que le corresponde dentro del grupo; para ello se propone la crítica ideológica y la aplicación de procedimientos del psicoanálisis que posibilitan la comprensión de la situación de cada individuo, descubriendo sus intereses a través de la crítica. El conocimiento se desarrolla mediante un proceso de construcción y reconstrucción sucesiva de la teoría y la práctica.

Entre las características más importantes del paradigma sociocrítico aplicado al ámbito de la educación se encuentran: a) la adopción de una visión global y dialéctica de la realidad educativa; b) la aceptación compartida de una visión democrática del conocimiento así como de los procesos implicados en su elaboración; y (c) la asunción de una visión particular de la teoría del conocimiento y de sus relaciones con la realidad y con la práctica, como lo referencia Alvarado y García (2008). Así, la idea de que la aplicación de los principios del paradigma sociocrítico como fundamento de las investigaciones - sean estas en el campo de la EA o en el de la enseñanza de las ciencias-, demuestra que es eficiente para generar cambios en las comunidades tanto si están en áreas socioeconómicamente problematizadas o no y hasta donde se ha experimentado, según los trabajos reportados, es de gran utilidad en ámbitos educativos con énfasis en sus problemas sociales y en las situaciones de enseñanza, aprendizaje y evaluación de asignaturas diversas.

Desde este punto de partida, la investigación cualitativa ofrece la posibilidad de interlocución con la compleja realidad de los sujetos que participan en el estudio, permite construir, resinificar y dialogar con la información obtenida con el objeto de establecer un panorama de lectura 
de la realidad abordada. Rescatando la importancia entre las relaciones de los sujetos, los contextos y la singularidad de sus experiencias como riqueza para realizar una construcción teórica (León, 2006).

En este sentido desde la metodología de la etnografía rápida (Clavijo y Páramo, 2006), se localizaron cuatro instituciones educativas que cumplieran con la premisa de ser diferentes en la naturaleza de los recursos económicos con los cuales determina su funcionamiento, por tanto se escogen dos instituciones privadas, una institución de concesión, es decir de carácter privado que en acuerdo con el Distrito presta el plantel y el cuerpo docente para estudiantes de estratos 1, 2, y 3 con referentes educativos de una educación pública, y una institución de carácter público.

La escogencia de las instituciones tuvo que ver con la disposición del grupo de maestros que allí laboran en la participación del estudio, ya que se parte de la idea del maestro como investigador, como sujeto que reflexiona sobre su quehacer pedagógico y toma decisiones acerca de problematizar el acto educativo en aras de fortalecer el proceso de aprendizaje y comprensión de sus educandos. En este sentido, la incorporación de la EA en el aula se convierte en el interés de un grupo de maestros que deciden participar en el análisis de los fundamentos didácticos y pedagógicos que conlleva una formación ambiental, interesados en ser escuchados acerca de cómo han llevado a cabo dicho trabajo, qué han obtenido y qué esperan al respecto. Son maestros de Ciencias Naturales de básica secundaria, que con su labor cotidiana han logrado incluir la dimensión ambiental en sus instituciones y que más allá de sus reflexiones individuales permitieron expresar sus sentires y experiencias frente a la EA.

La técnica de recolección de información se realizó a través de los grupos focales (Errandonea, 1997), la cual consiste en la producción de significados que apuntan a la indagación e interpretación de fenómenos ocultos a la observación de sentido común. Además, el método se caracteriza por trabajar con instrumentos de análisis que no buscan informar sobre la extensión de los fenómenos (cantidad de fenómenos), sino más bien interpretarlos en profundidad y detalle, para dar cuenta de comportamientos sociales y prácticas cotidianas. Por tanto se realizó una sesión de encuentro en el que se compartieron las experiencias de los maestros y se resolvieron cada uno de los cuestionamientos acerca de la forma de llevar la EA al aula de clase, lo que es consecuente con la etnografía rápida ya que el investigador reúne a los sujetos de estudio y en un corto tiempo desencadena una situación de riqueza de saberes frente al cuestionamiento de la EA, en este caso.
La investigación tuvo las siguientes fases que orientaron el marco de posibilidad de ejecución de los objetivos planteados:

Fase 1. Caracterización. Incluyó la identificación de los criterios conceptuales, metodológicos y operativos desde el docente en las cuatro instituciones educativas, con lo cual se reconocieron las posibilidades e imposibilidades de la incorporación de la EA en la escuela; luego esta misma identificación se realizó desde el documento de la PNEA.

Fase 2. Indicadores evaluativos. Se definieron los indicadores de evaluación según los criterios de posibilidad e imposibilidad resultantes de la comparación entre la experiencia de los maestros y la PNEA.

Fase 3. Estrategia evaluativa. Se construyó como propuesta inicial de evaluación con los indicadores de posibilidad e imposibilidad de la incorporación de la EA en la escuela, validando y ajustando la recopilación de la información.

\section{Resultados y análisis}

\section{Fase 1. Caracterización}

Se realizó un grupo focal que tuvo lugar en febrero de 2009, con un equipo de 12 profesores del área de Ciencias Naturales, pertenecientes a cuatro instituciones educativas diferentes de la ciudad de Bogotá, interesados en la discusión acerca del proceso de incorporación de la EA en la escuela. En el cuadro 1 se evidencian tres de los cuestionamientos realizados y tres respuestas de los participantes en el grupo focal. 


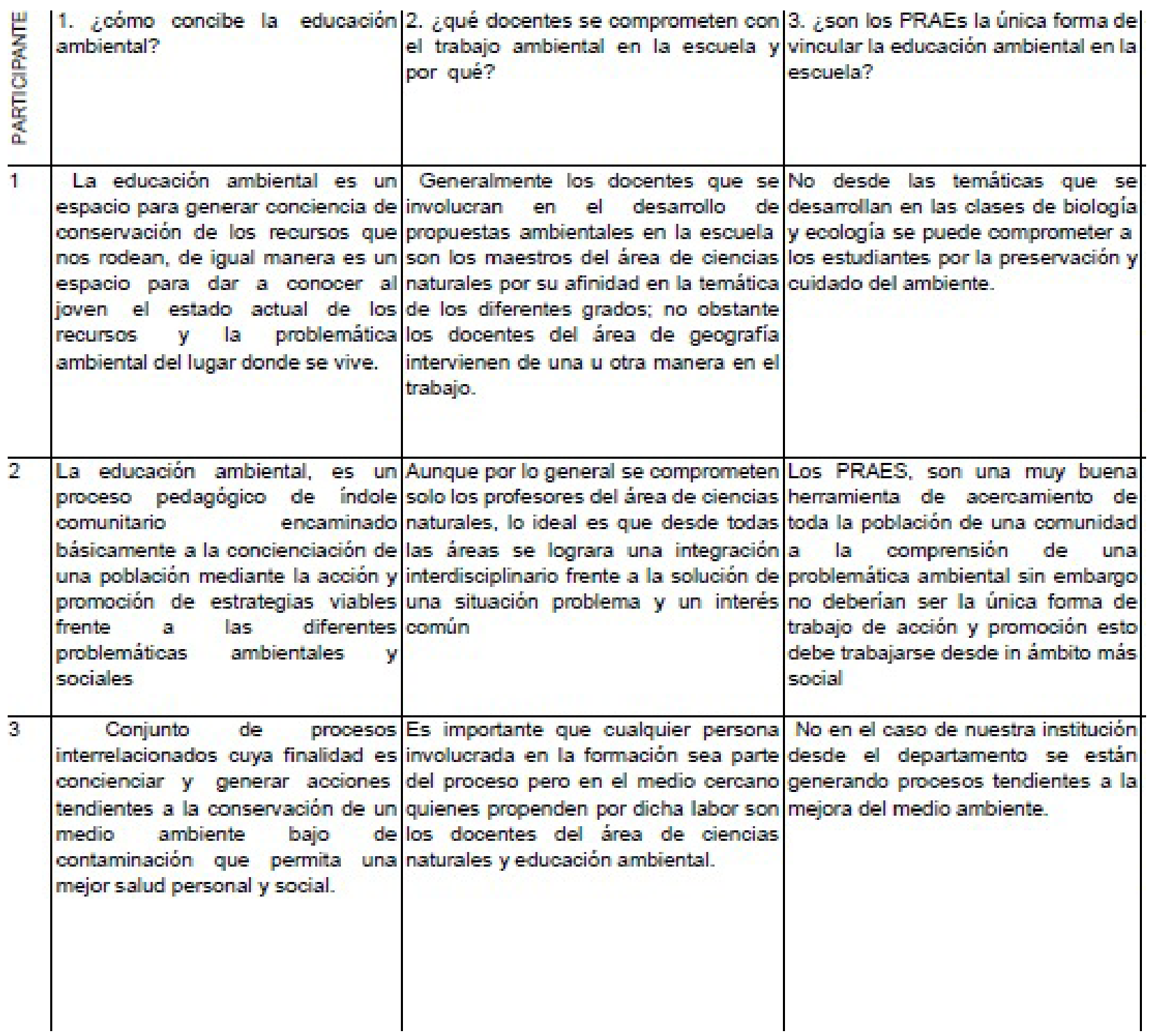

Cuadro 1. Respuestas al grupo focal.

El diseño de los cuestionamientos orientadores se realizó con el objetivo de indagar acerca de concepciones, procesos metodológicos y operativos que se siguieron por parte de los maestros, de acuerdo con su contexto escolar, en la puesta en marcha de la EA con la comunidad educativa.

Desde el análisis y lectura del documento de la PNEA, se determinaron como categorías los lineamientos propuestos que responden a los conceptos centrales que se deben trabajar en la transversalidad del currículo como lineamientos conceptuales, determinando las definiciones que en el texto se señalan se deben aprender y enseñar, en este caso los lineamientos conceptuales fueron: ambiente, educación ambiental, investigación y escuela.
Según el texto de la PNEA, los lineamientos metodológicos responden a las formas de viabilizar la enseñanza de los conceptos centrales a trabajar en educción ambiental, estos son: PRAE, grupos ecológicos y formación ambiental. Y por último los lineamientos operativos que define el texto como los que hacen posible que los lineamientos metodológicos tengan oportunidad de darse en la infraestructura escolar, estos son: tiempo, currículo y financiación.

El cruce de información entre las respuestas dadas por el grupo focal y los lineamientos de la PNEA se obtiene a través de una matriz de resultados que permite evidenciar la experiencia de los maestros con lo que dicta el documento. 
Ambiente. En esta categoría se logra evidenciar cómo mientras en el texto de la PNEA se conceptualiza el ambiente como un sistema dinámico con interacciones, los maestros no reconocen las interacciones presentes sino el valor extrínseco del ambiente como medio, entorno y recurso, como concepción y desde la relación del accionar del hombre sobre lo natural; por tanto define la forma de acercarse al estudio ambiental en la escuela desde la teoría, el concepto y lo que se dice allí de su cuidado como recurso. Premisa opuesta a los objetivos de la PNEA, ya que asegura que la comprensión del ambiente como sistema dinámico e interactuante es clara y por tanto se lograría pasar a establecer las relaciones ambientales responsables entre el sistema social y natural (Gutiérrez, 1993).

Educación ambiental (EA). Las concepciones sobre la EA se diversifican, desde la PNEA se toma como proceso para la comprensión de relación hombre/entorno; desde el quehacer del maestro se toma como un espacio, un proyecto desde la institución enfocado a resolver problemas, y finalmente coinciden en el proceso integral. Pareciera entonces que la EA en la escuela se está entendiendo desde diversos escenarios de aprendizaje y construcción que, aunque parecen apuntar al mismo objetivo, pueden llegar a confundir o a permitir un todo metodológico.

La diversidad de concepciones frente a la EA del maestro logra confundir los objetivos propuestos por el documento de la PNEA en el sentido de la realización de actividades, clases, campañas, consultas y ejercicios de investigación se asemejen a la apropiación de una realidad ambiental. De esta forma se termina por reconocer la temática ambiental como un activismo suelto sin reflexión y sentido de formación integral para el estudiante.

Investigación. Desde la concepción del maestro, la investigación parece tornarse como un elemento que contribuye a responder de forma precisa las inquietudes que surgen en el ámbito educativo, como una fuente de conocimiento en donde si hay tiempo se complejiza; si no, se realizan actividades que encaminan su desarrollo.

Por otro lado, la PNEA habla de un proceso complejo que requiere de tiempo y constante evaluación retroalimentada que parece no estar de acuerdo con la realidad de la escuela. De esta forma, en la escuela la investigación pasa a ser un ejercicio de consulta con miras a aproximarse a un proceso riguroso en donde se plantee desde el contexto un análisis de la realidad, no obstante el ejercicio de investigación se convierte en una metodología científica que cumple con el objetivo de reconocer un problema aparente, una temática, y presentar unos resultados de cambio inmediato en un determinado lugar (Colon y Melich, 1997).

Escuela. La PNEA plantea una escuela participativa y contextualizada; no obstante, dentro del contexto del sistema educativo colombiano un factor que va en contravía de tal premisa es el de la presión por el cumplimiento a cabalidad del currículo, que es tal vez señalado por los maestros al referirse como norma y límite hacia la escuela, en donde la participación está mediada por los fines y demandas específicas del sistema educativo. Por ejemplo el cumplimiento del PRAE en todas las instituciones escolares que pueden o no posibilitar otros factores de aprendizaje ambiental pero que independientemente de este fin, se debe gestar en los tiempos y particularidades de la comunidad educativa. Lo que conlleva a determinar de forma urgente el desarrollo del PRAE a partir de unas obligaciones imprescindibles -entre otras, el contenido curricular del año lectivo escolar-, lo que no deja tiempo ni espacio para una construcción participativa, activa, interdisciplinar y con sentido de la comunidad escolar.

\section{Lineamientos metodológicos}

PRAE. Desde la PNEA se comprenden los PRAE como proyectos de investigación que posibilitan la comprensión de una problemática ambiental; mientras que para los maestros es una estrategia de comprensión, de realización, una oportunidad de aprendizaje de la realidad ambiental y una norma que se debe cumplir. Es evidente que para algunos se ha convertido en la forma más clara de incorporar la EA en la escuela y en la única estrategia clara de consecución de resultados de impacto ambiental efectivo y socializable dentro de la comunidad escolar. Esto ha producido además que tan solo con desarrollar una temática del PRAE se considere abordado e incorporado el tema ambiental desde el concepto y la metodología en la escuela.

Grupos ecológicos. La estrategia de grupos ecológicos es nombrada por los maestros como una forma casi secundaria que deriva de la puesta en marcha de proyectos educativos y que, incluso, se centra en otro tipo de reflexiones sobre el ambiente.

Por otro lado, en la PNEA parece ser una oportunidad de profundizar en el estudio y abordaje de la temática ambiental, pero en la escuela no parece tener un protagonismo esencial. Esto debido tal vez a que la urgencia por tratar temas ambientales dese el PRAE puede producir una motivación a corto plazo por parte de los estudiantes, que no trasciende más allá de la realización de una actividad y por tanto no se considera necesario la conformación de un grupo ecológico que dé continuidad al tema.

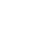


Formación ambiental. La PNEA define la formación ambiental casi desde el cambio en la escala de valores y las actitudes preferencialmente; mientras que los maestros diversifican su comprensión de la formación, en los aspectos propios de las clases de ciencias, todas las actividades que se realizan y el fundamento pedagógico que lo sustenta, pareciera obtenerse una visión más detallada, real y acorde desde la escuela que desde la PNEA. Lo que indica que en la formación ambiental que se está adelantando en la escuela interviene una diversidad de objetivos, propósitos, sentidos, aprendizajes, que pueden confundirse en la orientación académica y quedar suspendida entre otra materia para enseñar y aprender equivalente a las otras que aborda la escuela.

\section{Lineamientos operativos}

Tiempo. Este aspecto no parece tener ninguna similitud, se contradice totalmente lo que define la PNEA con lo encontrado por los maestros en su quehacer; mientras la primera exige que el tiempo sea flexible, los otros (en el contexto escolar) definen los límites y las dificultades de realización de actividades académicas por su inexistencia. Esta realidad ha determinado la profundidad del desarrollo de lo ambiental dentro de la comunidad, convirtiendo la EA en una actividad de un día o dos que se puede incluir en un acto protocolario escolar; o si no se permite dentro de la jornada académica, se busca la participación voluntaria en horas y espacios diferentes que terminan por defender una concepción de la EA fuera de la escuela.

Currículo. Este aspecto presenta inconsistencias importantes, ya que en el sistema educativo el pilar es el currículo. La PNEA por un lado plantea su flexibilidad y dinámica, mientras que la vivencia de los maestros demuestra que desde su concepción las clases de Ciencias y los proyectos de aula pasan por una rigidez que no permite desarrollo en tiempos y espacios más allá de los dados por el PRAE, de forma aislada entre áreas disciplinares y sin posibilidad de encuentros entre pares académicos o la comunidad educativa para su construcción o reflexión constructiva. Así, se establece que la forma pertinente en que la escuela aborda la EA, desde el currículo, es con el desarrollo del PRAE y las clases cátedra de Ecología y EA propiamente.

Financiación. Al parecer, la PNEA tiene claro las entidades de apoyo económico para la realización de los proyectos ambientales; no obstante los maestros en su quehacer, no nombran otro agente más que la comunidad y la intervención de esta en el PRAE, limitando su hacer a lo que se posibilite desde los recursos de la comunidad única- mente. Esto se convierte en un obstáculo para la realización de procesos dentro del abordaje ambiental que a su vez determina que la única incorporación de la EA en la escuela -o la más viable- es de forma teórica.

\section{Fase 2. Indicadores evaluativos}

Se elaboró un panorama de análisis dado por el grupo focal de maestros y el documento de la PNEA que definió los indicadores que median entre lo deseado -como panorama propuesto por el documento de la PNEA-, y la realidad -vivenciada por los maestros en la escuela-, sobre la puesta en marcha de la EA, y definen la posibilidad de su incorporación, así como los elementos que aún no se evidencian en el proceso y, por tanto, hacen que no se dé una incorporación real de la EA en la escuela.

Se construyeron enunciados que posibilitaban o no una acción educativa en la escuela desde la enseñanza de los lineamientos conceptuales, el abordaje de los lineamientos metodológicos y los operativos en su puesta en marcha en la escuela, fueron discutidos y retroalimentados desde las diversas posturas de asumir la enseñanza ambiental con los maestros en el aula, con lo cual se conoció qué se lograba y qué no era posible desde la experiencia educativa. Por ejemplo, para el concepto de ambiente se construyeron los indicadores señalados en el cuadro 2. 


\begin{tabular}{|c|c|c|c|}
\hline Indicador & Posibilidad & Imposibilidad & Discusión \\
\hline $\begin{array}{l}\text { Es abordado en las clases } \\
\text { solamente desde la funda- } \\
\text { mentación teórica de las } \\
\text { Ciencias Naturales como } \\
\text { objeto de estudio. }\end{array}$ & $\begin{array}{l}\text { Aprendizaje del ambiente } \\
\text { como concepto escolar. }\end{array}$ & $\begin{array}{l}\text { Comprensión del reconoci- } \\
\text { miento de las prácticas y de } \\
\text { la realidad de existencia del } \\
\text { ambiente. }\end{array}$ & $\begin{array}{l}\text { Se aprende desde la teoría como concepto } \\
\text { escolar que debe abordarse, haciendo que se } \\
\text { limite la comprensión y no se dé el reconoci- } \\
\text { miento y la apropiación de la existencia de su } \\
\text { ser en un ambiente con el que interacciona } \\
\text { cotidianamente (Sauvé, 1994). }\end{array}$ \\
\hline $\begin{array}{l}\text { Es abordado en el aula } \\
\text { desde la problemática } \\
\text { como efecto de la mala } \\
\text { relación hombre/natura- } \\
\text { leza. }\end{array}$ & $\begin{array}{l}\text { De motivación por la inda- } \\
\text { gación del estado ecológico } \\
\text { del ambiente y sus posibles } \\
\text { soluciones. }\end{array}$ & $\begin{array}{l}\text { En el reconocimiento de la } \\
\text { no supremacía del hombre } \\
\text { frente al ambiente natural, y } \\
\text { de la construcción cultural, } \\
\text { política y económica como } \\
\text { sistemas influyentes. }\end{array}$ & $\begin{array}{l}\text { Si se analiza el ambiente desde el problema } \\
\text { por solucionar, puede darse una aproxima- } \\
\text { ción a los efectos que el hombre ha causado, } \\
\text { así como la posibilidad de la solución desde e } \\
\text { individuo, pero como colectivo social de cons- } \\
\text { trucción económica y política puede descono- } \\
\text { cerse su accionar, así como la dinámica propia } \\
\text { del sistema ambiental (Sauvé, 1996). }\end{array}$ \\
\hline $\begin{array}{l}\text { Es abordado en el aula } \\
\text { como un eje transversal de } \\
\text { conocimiento y de interac- } \\
\text { ción entre las dinámicas } \\
\text { sociales y naturales. }\end{array}$ & $\begin{array}{l}\text { De orientar el proceso edu- } \\
\text { cativo desde la integración } \\
\text { de las disciplinas, que nutra } \\
\text { la comprensión de la com- } \\
\text { plejidad de relaciones que } \\
\text { se desarrollan. }\end{array}$ & $\begin{array}{l}\text { De lograr espacios y } \\
\text { momentos de encuentros } \\
\text { en la dinámica de tiempo, } \\
\text { currículo y actores en la } \\
\text { escuela. }\end{array}$ & $\begin{array}{l}\text { Es posible considerar el ambiente como un } \\
\text { eje transversal de conocimiento pero es impo- } \\
\text { sible de abordarse de esta forma desde la } \\
\text { dinámica escolar, por tanto no se logra com- } \\
\text { prender en conjunto si no como fragmentos } \\
\text { sin coherencia (Gutiérrez, 1993). }\end{array}$ \\
\hline $\begin{array}{l}\text { Es entendido como medio } \\
\text { ambiente en la comunidad } \\
\text { escolar. }\end{array}$ & $\begin{array}{l}\text { Reproducir el error con- } \\
\text { ceptual que conduce a una } \\
\text { comprensión descontextua- } \\
\text { lizada del ambiente. }\end{array}$ & $\begin{array}{l}\text { En el reconocimiento del } \\
\text { ambiente como el medio } \\
\text { en el cual interaccionan } \\
\text { diversos sistemas de orga- } \\
\text { nización cultural, social y } \\
\text { natural en una dinámica de } \\
\text { continua transformación. }\end{array}$ & $\begin{array}{l}\text { Si no se logra comprender que el sentido y sig- } \\
\text { nificado de los conceptos medio y ambiente } \\
\text { son complementarios, pero que divergen en } \\
\text { la forma de concebir las prácticas de rela- } \\
\text { ción, no se logrará establecer un fundamento } \\
\text { teórico en el discurso ambiental que oriente } \\
\text { satisfactoriamente las acciones y relacio- } \\
\text { nes entre los sujetos en el ambiente (Angulo, } \\
\text { 1995). }\end{array}$ \\
\hline $\begin{array}{l}\text { Es abordado teórica y } \\
\text { prácticamente dese las } \\
\text { diversas corrientes de pen- } \\
\text { samiento en la formación } \\
\text { de los maestros. }\end{array}$ & $\begin{array}{l}\text { En la incorporación en la } \\
\text { escuela de todas las concep- } \\
\text { ciones, modalidades, creen- } \\
\text { cias y formas de concebir el } \\
\text { ambiente. }\end{array}$ & $\begin{array}{l}\text { En la orientación en la forma } \\
\text { adecuada, pertinente o } \\
\text { satisfactoria de definir una } \\
\text { actividad, fundamento teó- } \\
\text { rico, concepción o práctica, } \\
\text { respecto al ambiente, por } \\
\text { tanto todo vale desde el } \\
\text { maestro que lo guie. }\end{array}$ & $\begin{array}{l}\text { La diversidad de formas de pensar y asumir e } \\
\text { ambiente, conlleva a que los maestros orien- } \\
\text { ten según su comprensión del tema y así se } \\
\text { desarrollen los fundamentos de enseñanza y } \\
\text { de prácticas ambientales, por tanto se puede } \\
\text { caer en un activismo, en un ambientalismo, } \\
\text { ecologismo, entre otras formas de pensar y } \\
\text { actuar frente al ambiente, en donde la pre- } \\
\text { gunta por el cómo y por el qué es EA se hace } \\
\text { necesaria de abordarse (Sauvé, 1994). }\end{array}$ \\
\hline
\end{tabular}

Cuadro 2. Indicadores evaluativos del concepto ambiente 
De esta manera se elaboraron los indicadores para cada uno de los lineamientos conceptuales, metodológicos y operativos que más adelante se convirtieron en el cuerpo de la estrategia evaluativa.

\section{Fase 3. Estrategia evaluativa}

Se propuso una estrategia evaluativa que contribuyera a que en la escuela el maestro confronte su hacer con las condiciones de posibilidad o de imposibilidad como panorama para la incorporación de la EA, con la intención de ofrecer otra lectura de la realidad que se da durante puesta en marcha de la formación ambiental, no con el objeto de identificar los niveles de logro, sino con el de realizar la invitación a una reconstrucción en la forma de asumir la enseñanza y la formación de sociedad.

Presentación. La mirada evaluativa pretendió definir, en el proceso seguido por la escuela, un panorama que contempló el escenario de posibilidad o de imposibilidad que la incorporación de la EA ha suscitado dentro del contexto particular. Por lo que a través de una escala valorativa (Ver tabla $n^{\circ} 2$ ) se situó el estado del proceso seguido en la escuela, desde las condiciones posibles e imposibles de cada categoría que indicó la forma como se ha apropiado la acción educativa.

Se presentaron por tanto tres lineamientos: conceptual, procedimental y operativo. En el conceptual, se definieron las categorías de: ambiente, EA, investigación y escuela.

Para el lineamiento metodológico se plantearon las categorías de: PRAE, grupos ecológicos y formación ambiental.

En el lineamiento operativo se incluyeron las categorías de: tiempo, currículo y financiación.

Para cada una de dichas categorías se expusieron de 4 a 8 indicadores que definieron diversas formas de concepción de la categoría que a su vez determinó qué posibilidad o imposibilidad presentó en el desarrollo en la escuela.

De esta forma el objeto fue que en la escala valorativa que va de 0 a 100, ubicada debajo de cada premisa de posibilidad e imposibilidad, luego de la lectura juiciosa, se ubicara un solo valor que representara el estado del proceso a llevar en la escuela (cuadro 3 ).

Una vez obtenido el puntaje total por cada categoría se entiende que si este puntaje fue menor a 50: el estado del proceso seguido para la incorporación de la EA en la escuela se ha desarrollado dentro de las condiciones de posibilidad; esto quiere decir que dentro de las pau- tas ideales propuestas por la PNEA, en la escuela, se ha incorporado la EA según la realidad que permitió que se dieran algunas condiciones de tipo conceptual, metodológico y operativo. No obstante, la invitación consistió en revisar si las condiciones de posibilidad favorecían una educación integral, así como la pertinencia y viabilidad del proceso, en miras de establecer el horizonte de sentido pedagógico en la proyección de una sociedad con responsabilidad ambiental.

Si el puntaje era mayor a 50: el estado del proceso seguido para la incorporación de la EA en la escuela se ha desarrollado dentro de las condiciones de Imposibilidad; esto quiere decir que dentro de las pautas ideales propuestas por la PNEA, en la escuela, se ha incorporado la EA de acuerdo con la realidad, lo que ha producido resultados que no satisfacen, permanecen o aportan a los desarrollos para una EA, y que por el contrario los lineamientos de tipo conceptual, metodológico y operativo no ofrecen escenarios viables y pertinentes para una incorporación ambiental contextualizada. La invitación fue precisamente a considerar cómo se lograría esa anhelada restructuración en la concepción y desarrollo del sistema educativo para que pueda brindar escenarios posibles de construir desde el contexto escolar real y particular, propiciando una educación integral con propuestas claras y posibles de asumir desde el quehacer del maestro, en miras de establecer el horizonte de sentido pedagógico en la proyección de una sociedad con responsabilidad ambiental. 
Cuadro 3. Estrategia evaluativa

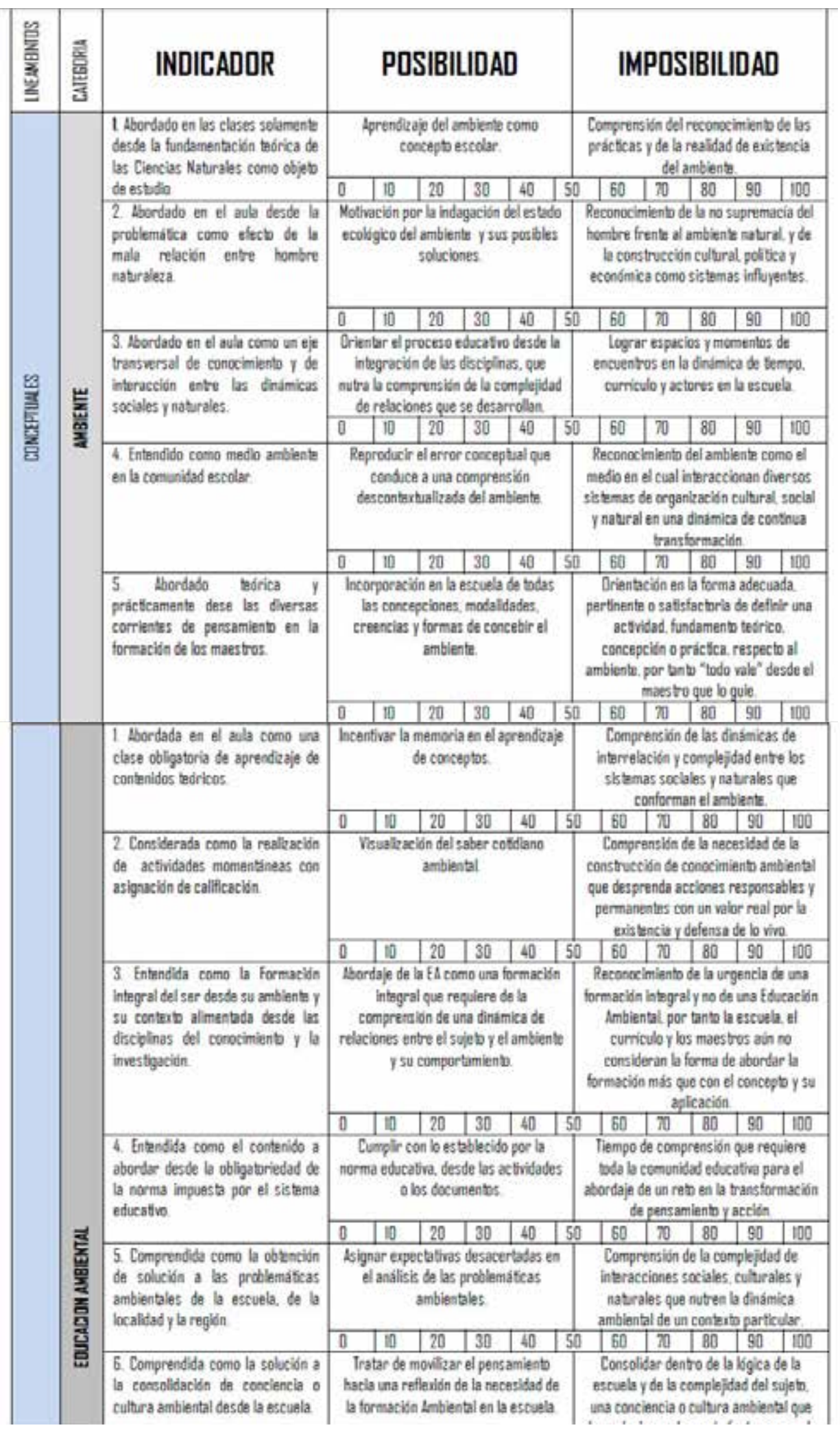




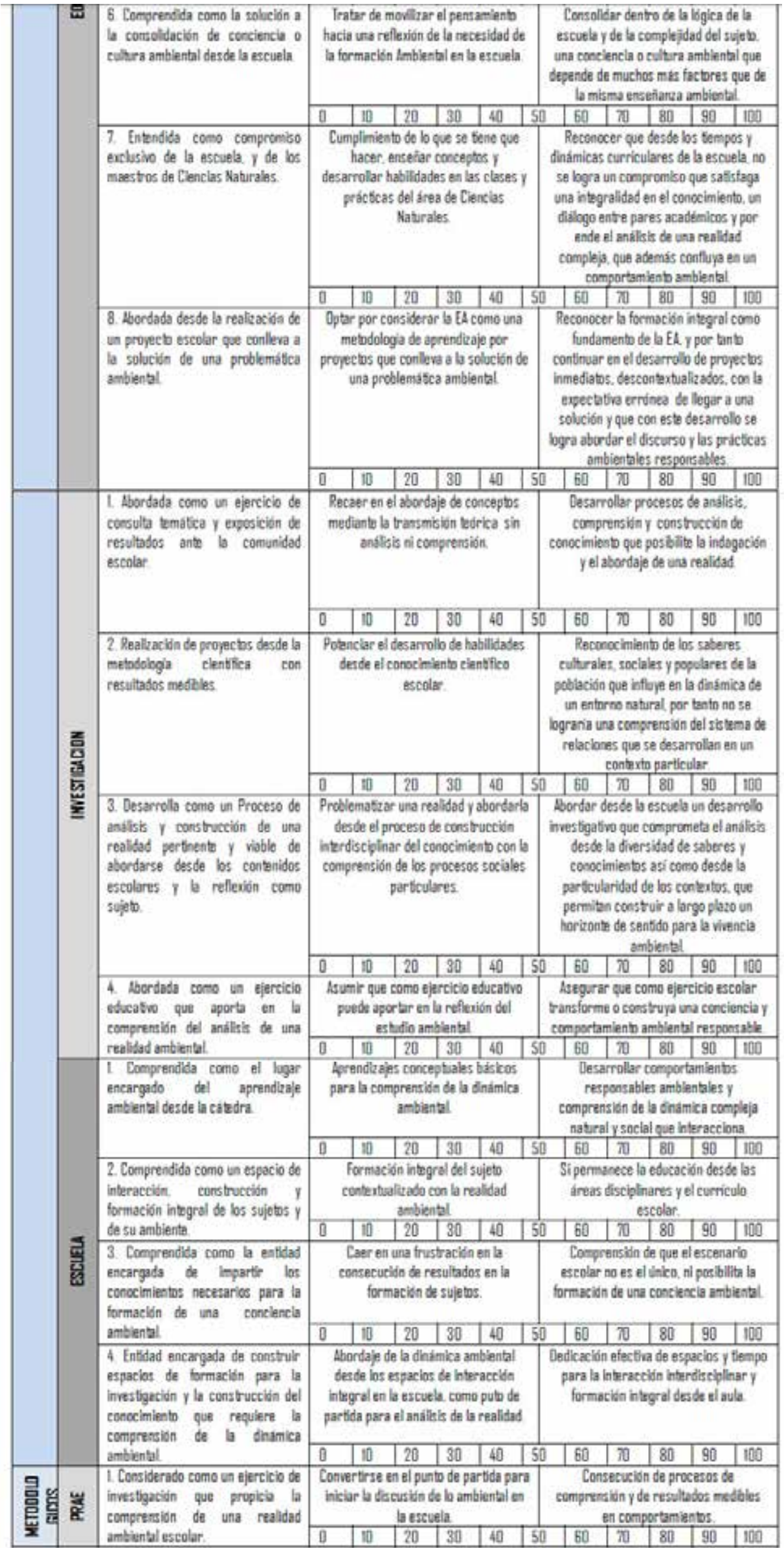




\begin{tabular}{|c|c|c|c|c|c|c|c|c|c|c|c|c|}
\hline & \multirow[t]{2}{*}{$\begin{array}{l}\text { 2. Considerado como un projecto } \\
\text { de investogación que soluciona las } \\
\text { problematicas amblentales de la } \\
\text { localdad escolar }\end{array}$} & \multicolumn{6}{|c|}{$\begin{array}{l}\text { Cenvertic la methdologia en una } \\
\text { práctea para solamiente la } \\
\text { consecucidn de soluciones } \\
\text { amblentzles. }\end{array}$} & \multicolumn{5}{|c|}{$\begin{array}{l}\text { Comprender el desarrollo de la } \\
\text { metndologia como un ejercicio de } \\
\text { acercamiento al sstudlo de una realidad } \\
\text { y a la proyeccien de posibles aportes a } \\
\text { las dinamicas de solución. }\end{array}$} \\
\hline & & 0 & 10 & 20 & 80 & 40 & 50 & 6 & 70 & 80 & 90 & 100 \\
\hline & \multirow[t]{2}{*}{$\begin{array}{l}3 \text { Considerado conio un project } \\
\text { ambiental que debe implementar la } \\
\text { estuela para aprender y } \\
\text { desamollar conciencla amblental }\end{array}$} & \multicolumn{6}{|c|}{$\begin{array}{l}\text { Confundlr los alcances de una } \\
\text { metodologia escolar con el ideal } \\
\text { educativa. }\end{array}$} & \multicolumn{5}{|c|}{$\begin{array}{l}\text { Reconocer que el comipromisa de } \\
\text { responsabildad ambiental conlleva mas } \\
\text { que la conciencia el cambio en la forma } \\
\text { le concebir el sistema de vida del sujetb } \\
\text { en la sociedad actual y que aquello } \\
\text { sucera el conocimiento escolar }\end{array}$} \\
\hline & & 0 & 10 & 20 & 80 & 40 & 50 & 16 & 70 & 80 & 90 & 100 \\
\hline & \multirow[t]{2}{*}{$\begin{array}{l}\text { 4. Considerado como la única forma } \\
\text { posible de aprender temas y } \\
\text { desamollar } \\
\text { concemientes con b ambiental } \\
\text { dentro de la escuela. }\end{array}$} & \multicolumn{6}{|c|}{$\begin{array}{c}\text { Consfturio como el escenario } \\
\text { gertinente dentro de la logica escolar. } \\
\text { de propiciar el abordaje de la dinámica } \\
\text { ambiental }\end{array}$} & \multicolumn{5}{|c|}{$\begin{array}{l}\text { Profunditación y coobutualitación de la } \\
\text { aprehensido de la realidad ambiental que } \\
\text { requiere el centerto y el sejab. }\end{array}$} \\
\hline & & 0 & 10 & 20 & 30 & 40 & 50 & E. & 70 & 80 & 90 & 100 \\
\hline \multirow{6}{*}{ 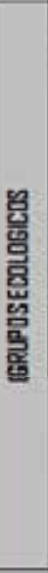 } & \multirow[t]{2}{*}{$\begin{array}{l}\text { 1. Comprendidos como estrategla } \\
\text { para protundiar en al estudio del } \\
\text { ambiente. }\end{array}$} & \multicolumn{6}{|c|}{$\begin{array}{l}\text { Fundamentar el compromico de } \\
\text { algunos en el estudio ambiental }\end{array}$} & \multicolumn{5}{|c|}{$\begin{array}{l}\text { Convertirse en estrabgia de } \\
\text { multelicadores del estudio ambiental ya } \\
\text { que precisamentu parts de un } \\
\text { compromitso partcular. y podria } \\
\text { convertirse en una socializacion de } \\
\text { resulbdos. }\end{array}$} \\
\hline & & 0 & 10 & 20 & 30 & 40 & 50 & \begin{tabular}{|l|l} 
& 8 \\
\end{tabular} & 70 & 80 & 90 & 100 \\
\hline & \multirow[t]{2}{*}{$\begin{array}{l}\text { 2. Comprendidos como el rasultado } \\
\text { del interés del aprendeje } \\
\text { ambiental escolar. }\end{array}$} & \multicolumn{6}{|c|}{$\begin{array}{l}\text { Propectarse como grupo de } \\
\text { inrestogación que coestione } \\
\text { construpa estudios ambienteles. }\end{array}$} & \multicolumn{5}{|c|}{$\begin{array}{l}\text { Considerados como logro de una EA } \\
\text { intgral escolar que perdure y resulbe en } \\
\text { ciudatanos responsables con el } \\
\text { ambient. }\end{array}$} \\
\hline & & 0 & 10 & 20 & 30 & 40 & 50 & 160 & $x$ & 80 & 90 & 100 \\
\hline & \multirow[t]{2}{*}{$\begin{array}{l}\text { 3. Enendidos como los } \\
\text { responsables de la comprention de } \\
\text { la realdad ambiental escolar. }\end{array}$} & \multicolumn{6}{|c|}{$\begin{array}{c}\text { Lbordar la Ė desde la asignacifio de } \\
\text { tareas y lideres dentro de la isea de la } \\
\text { Poltica Ambiental. }\end{array}$} & \multicolumn{5}{|c|}{$\begin{array}{l}\text { Comprometer la comunidad educativa en } \\
\text { la particularidad de los interese de can } \\
\text { grupo. y asi misno quodería suelta en } \\
\text { sentoo y significado la formación } \\
\text { integrai en la escuela }\end{array}$} \\
\hline & & 0 & 10 & 20 & 30 & 40 & 50 & 6 & 70 & 80 & 90 & 100 \\
\hline \multirow{6}{*}{ 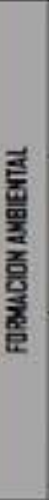 } & \multirow[t]{2}{*}{$\begin{array}{l}\text { 1. Comprendida como la cibedra de } \\
\text { fcologia con el desarrollo de } \\
\text { actuidades prictlcas que } \\
\text { desarrollen conciencla amblental }\end{array}$} & \multicolumn{6}{|c|}{$\begin{array}{l}\text { Confundir la feolegia con la EL en las } \\
\text { prácficas y fandamentos } \\
\text { eplistemoliglcos. Por b tanto serd una } \\
\text { materia mas que aprender. }\end{array}$} & \multicolumn{5}{|c|}{$\begin{array}{c}\text { Lsumir la formacioin integral desde la } \\
\text { complejidad de intaracciones sociales y } \\
\text { naturales }\end{array}$} \\
\hline & & D & 10 & 20 & 30 & 40 & 50 & 16 & $\pi$ & 80 & 90 & 100 \\
\hline & \multirow[t]{2}{*}{$\begin{array}{l}\text { 2. Comprendida como el desarrollo } \\
\text { de actividades que conlleven a la } \\
\text { ecesecucioin de cultura ambiental } \\
\text { escolar. }\end{array}$} & \multicolumn{6}{|c|}{$\begin{array}{c}\text { Coovertir la EA en un activismo sin } \\
\text { senbido ni direcciśn mas que el de la } \\
\text { urgencia por la evidencia de un logro } \\
\text { ambiental. }\end{array}$} & \multicolumn{5}{|c|}{$\begin{array}{l}\text { Concepeion de formacion letegral del } \\
\text { sujpte. }\end{array}$} \\
\hline & & D. & 11] & 20 & 30 & 40 & 50 & 6 & 西 & 80 & 80 & 100 \\
\hline & \multirow[t]{2}{*}{$\begin{array}{l}\text { 3. Comprendida come ol espacio de } \\
\text { refleuldn para la construccilin de } \\
\text { estrateglas de comprensión y } \\
\text { accín ambiental escolar }\end{array}$} & \multicolumn{6}{|c|}{$\begin{array}{l}\text { Consolidación de una formación } \\
\text { integral del sujeb que permila al } \\
\text { dialogo de saberes y desarrollos } \\
\text { tendientes a la transformación de la } \\
\text { incorporacido amblental en la escuela }\end{array}$} & \multicolumn{5}{|c|}{$\begin{array}{l}\text { Consecucife de cambios imefiatos en } \\
\text { las acitudes del sujetn }\end{array}$} \\
\hline & & 0 & 10 & 20 & 30 & 40 & 50 & ह1 & $\pi$ & 80 & 90 & 100 \\
\hline
\end{tabular}




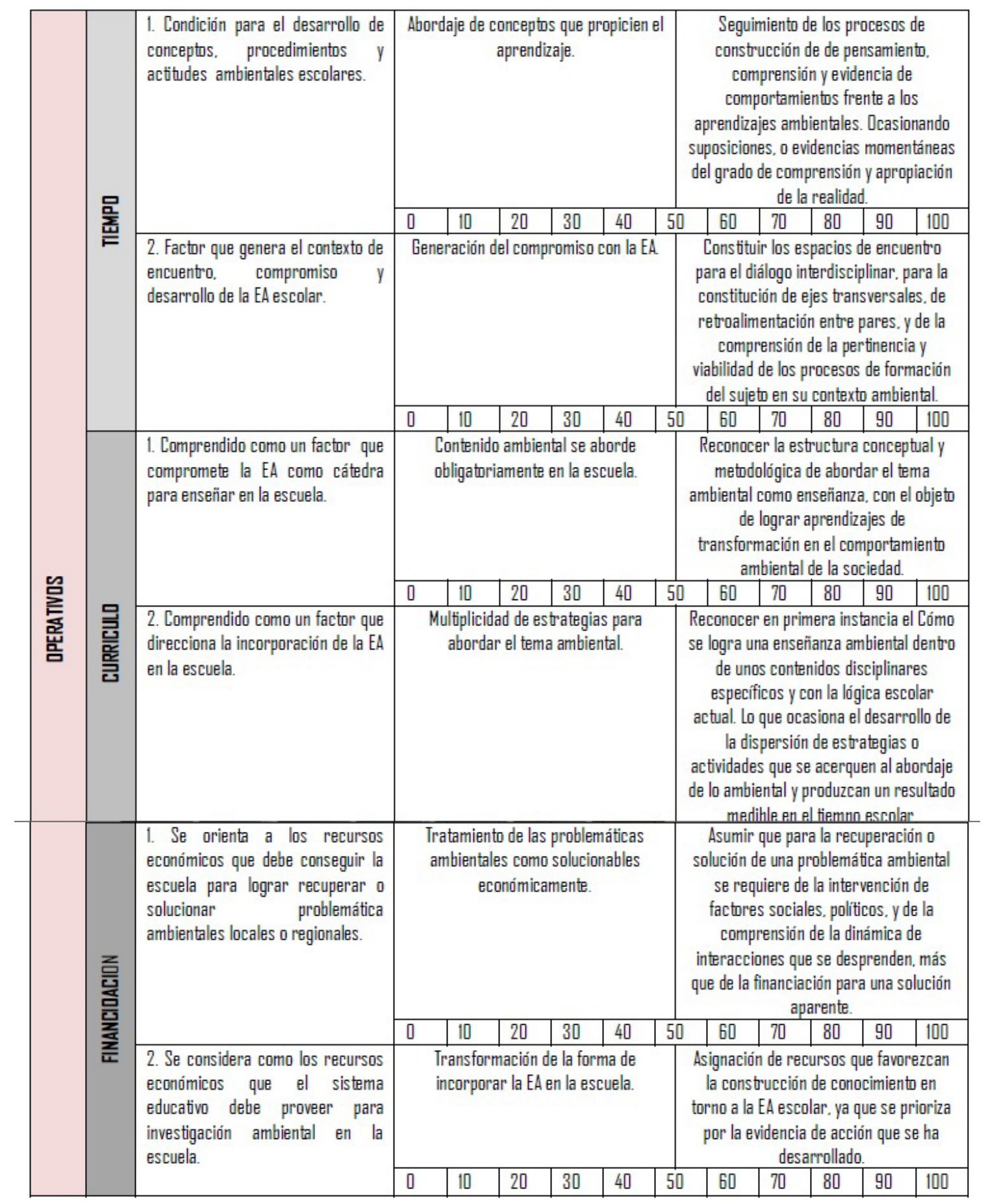




\section{Conclusiones}

El saber ambiental en la escuela está orientado desde la necesidad por el hacer por un activismo acelerado que intenta, más allá de la comprensión de las dinámicas ambientales, minimizar el desconocimiento del daño ambiental y propender por un estado de conservación de los recursos, en donde se expone la relación con el concepto de ambiente, como recurso para utilizar y proteger.

El conocimiento pedagógico es dado desde la visión del maestro que orienta el proceso dentro y fuera del aula, y en este caso, la urgencia por hacer evidente la formación ambiental; permite que se coloquen otros elementos del discurso ambiental que terminan por sectorizar una forma de comprender la EA como problema a resolver, como un espacio de formación, que conlleva a una formación más completa y compleja del individuo, una integral, que da cuenta de los valores ciudadanos en relación con el ambiente.

Por obligación, ley o formación, la EA sigue destinada al profesor de Ciencias Naturales y sigue enmarcada a sus formas metodológicas y construcciones epistemológicas, por ello, es que tal vez no se logran evidencias en el cambio de actitud y pensamiento, porque se aprendió a dar resultados para cada caso, no para proyectar los resultados más allá de las variables de estudio.

Los maestros han abordado el estudio ambiental desde la construcción del PRAE como elemento transversal, lo que reduce el campo de las estrategias metodológicas y operativas al diseño y ejecución de un solo proyecto. Lo que tal vez posiciona el conocimiento ambiental desde la recuperación, protección, y resolución de problemáticas ambientales locales a través de momentáneas actividades ecológicas en las que la comunidad participa sin comprensión, solo por cooperación con el ambiente.

La claridad conceptual y metodológica que se tiene del documento de la PNEA es contradictoria, ya que aunque es evidente que los principios básicos son nombrados y trabajados por los maestros; no obstante, en el momento de plantear las estrategias de consecución parecen alejarse del texto y tomar otros caminos metodológicos que plantean un momentáneo éxito y no la trascendencia de la formación.

La multiplicidad en las posturas frente al reconocimiento y estrategias de EA incurren en que los maestros, desde su comprensión y formación, diversifiquen la forma de abordar esta temática desde el activismo, la conservación, la investigación sin intervención, la sensibilización, la preocupación y la documentación, entre otras, lo cual termina en que cada estudiante comprenda y reconstruya concepciones y actitudes diversas y a veces contradictorias sobre la EA y el comportamiento responsable con el ambiente.

\section{Referencias bibliográficas}

Alvarado, L. y García, M. (2008). Características más relevantes del paradigma sociocrítico: su aplicación en investigaciones de educación ambiental y de enseñanza de las ciencias realizadas en el Doctorado de Educación del Instituto Pedagógico de Caracas. Sapiens, 9(2), 187-202.
Angulo, F. (1995). Volver a pensar la educación. Vol. II. Madrid: Morata/Paideia.

Arnal, J. (1992). Investigación educativa. Fundamentos y metodología. Barcelona: Labor.

Cano, E. (1998). La evaluación de la calidad de los sistemas educativos. En: E. Cano. Evaluación de la calidad educativa. Madrid: Editorial La Muralla.

Castro, J. (1997). Breve Historia de la Educación Ambiental. España: Alcántara.

Colom, A. y Melich, J. (1997). Después de la modernidad: nuevas filosofías de la educación. Barcelona: Ediciones Paidós.

Clavijo, A. y Páramo, P. (2006). Pedagogía urbana: Principios, aplicaciones y desafíos. Bogotá: Universidad Pedagógica Nacional.

Errandonea, A. (1997). ¿Metodología cualitativa versus metodología cuantitativa? Montevideo: Cuadernos de Clacso.

Gutiérrez, J. (1993). Enfoques teóricos en pedagogía ambiental: hacia una necesaria fundamentación epistemológica de las prácticas ecológicoeducativas. Revista de Educación de la Universidad de Granada, 7, 59-71.

León, A. (2006). La investigación cualitativa en educación. Lineamientos técnicos para el diseño del Programa Estatal de Integración Educativa. Documento inédito. Chiapas, México.

Maya, A. (1992). Ambiente y desarrollo. En: C. Quiroz (ed.). Ambiente y planificación. Un enfoque para el desarrollo hacia el siglo XXI. Bogotá: Secab.

Morin, E. (1999). Los siete saberes necesarios para la educación del futuro. París: Unesco.

Otero, E. (2006). Antecedentes de una propuesta: Hacia la educación ambiental. Manizales: Universidad de Caldas.

Ministerio del Medio Ambiente y Ministerio de Educación Nacional (2002). Política Nacional de Educación Ambiental (SINA). Bogotá.

Sauvé, L. (1994). Exploración de la diversidad de conceptos y de prácticas en la Educación Relativa al Ambiente. En: Memorias Seminario Internacional la Dimensión Ambiental y la Escuela. Bogotá: Ministerio de Educación Nacional.

Sauvé, L. (1996). La educación ambiental: hacia un enfoque global y crítico. En: Seminario Internacional de Investigación-Formación EDAMAZ. Montreal: Universidad de Quebec. 